\title{
Software benchmark design and use
}

\author{
Maya Daneva \\ Institute of Business Information Systems \\ at the University of Saarland \\ Altenkesseler str. 17, Geb.B2, D - 66115 Saarbrücken, GERMANY \\ phone:++49 (0681) 9762 224, fax: ++49 (0681) 77516 \\ E-mail:maya@iwisel.iwi.uni-sb.de
}

\begin{abstract}
This paper presents a holistic view of software benchmarking. We elucidate the concepts of the problem area by studying relevant benchmarking aspects like why software products are benchmarked, what benchmark is useful, how the benchmarks can be designed and used, and how the benchmarking process can be modelled and supported by means of software tools.
\end{abstract}

\section{Keywords}

Software benchmark, benchmark design, computer-aided software benchmarking

\section{INTRODUCTION}

The software community characterizes the performance of the marketed products in a variety of confusing metrics. The confusion is due to a lack of quantifiable performance information (Ghezzi et al., 1991), accurate models of software workload profile (McDonell, 1995), comprehensible software benchmark design methodology (Maneva et all., 1994) and credible interpretation rules (Gray, 1993).

In this paper we try to overcome these difficulties by proposing an unifying approach to software benchmark design and use. To ensure the feasibility of this approach, we develop the notion of computer-aided software benchmarking. 


\section{INTEREST IN SOFTWARE BENCHMARKING}

To develop an approach to software benchmark design we selected as a starting point the analysis of goals the organizations try to achieve by running benchmarks. This is used to characterize the useful software product benchmarks and to classify them.

Software products are benchmarked by the following classes of organizations each of which follows a different set of goals:

- Software developers: To uncover software bottlenecks and optimize software processes.

- Software distributors: To highlight strengths of the marketed products.

- Certification offices: To check a compliance between the required and the actual product.

- Software testing laboratories: To evaluate user satisfaction with the available software.

- Marketing agencies: To determine the market power of software products.

- Commercial computer magazines: To promote or to position a certain software product.

- Software researchers: To control software development through benchmarking.

- Academic institutions: To select the most appropriate software for a given project.

- Independent benchmarking companies: To learn and announce the truth to the user.

\section{CRITERIA TO SOFTWARE PRODUCT BENCHMARKS}

To be useful a software product benchmark should meet six important criteria:

- Pertinence: It must be closely related to customer's business problem.

- Functional coverage: It must be chosen so as to involve the software functionalities the user expects to use most in the system.

- Understandability: If it is complex to be understood, it will lack credibility (Gray, 1993).

- Ease of use: The benchmarking procedure should be relatively simple so as the costs including design time, hardware resources, and lost opportunity are optimized.

- Interpretability: The software product benchmark must provide decision rules for identifying the best software product among the competitors.

- Reproducibility: Each time the benchmark is run the results should remain unchanged.

\section{CLASSIFICATION OF SOFTWARE PRODUCT BENCHMARKS}

We suggest to group the software product benchmarks in two classes - generic and optional. By analogy to hardware system ratings, we consider the performance and the price/performance ratio as generic benchmarks. We follow the software engineering practice to equate performance with efficiency (Ghezzi et al., 1991). A software system is efficient, if it uses the computing resources economically.

To involve other product characteristics in benchmarking, we propose to use optional software benchmarks which fall into two classes - application-neutral and applicationspecific. The neutral benchmarks are constructed on the base of software domain-independent characteristics, for example: reliability, quality of documentation, ease of use, etc. The application-specific benchmarks are a response to the variety of software product uses, and thus, they are closely related to the domain the software is created for. For example, Set Query Benchmark is used for decision support systems, Cattel Benchmark - for CASE and CAD tools (Gray, 1993), and BECAUSE benchmarks - for compilers (Porscher, 1995).

Since a software characteristic can be defined by a set of metrics, it is reasonable to differentiate between elementary and complex benchmarks. A benchmark is elementary if a predefined value exists in a form of a software characteristic. A benchmark is complex, if it is 
determined from subordinate benchmarks. For example, data base resource utilization for a query (Set Query Benchmark) is defined by three elementary benchmarks: elapsed time, CPU time and Input/Output use (Gray, 1993).

Finally, the benchmarks can be differentiated on the base of its granularity, i.e. in dependence on what software aspect they refer to. For example, the benchmarks for a data base software might concern the whole data base, the data block, the record or the field.

\section{PROCEDURE FOR SOFTWARE BENCHMARK DESIGN AND USE}

After analyzing some benchmarking practices conducted by the worldwide known computer associations Standard Performance Evaluation Corporation, Transaction Processing Performance Council and Business Application Performance Consortium (Gray, 1993), we suggest the following procedure for software benchmark design and use (Figure 1):

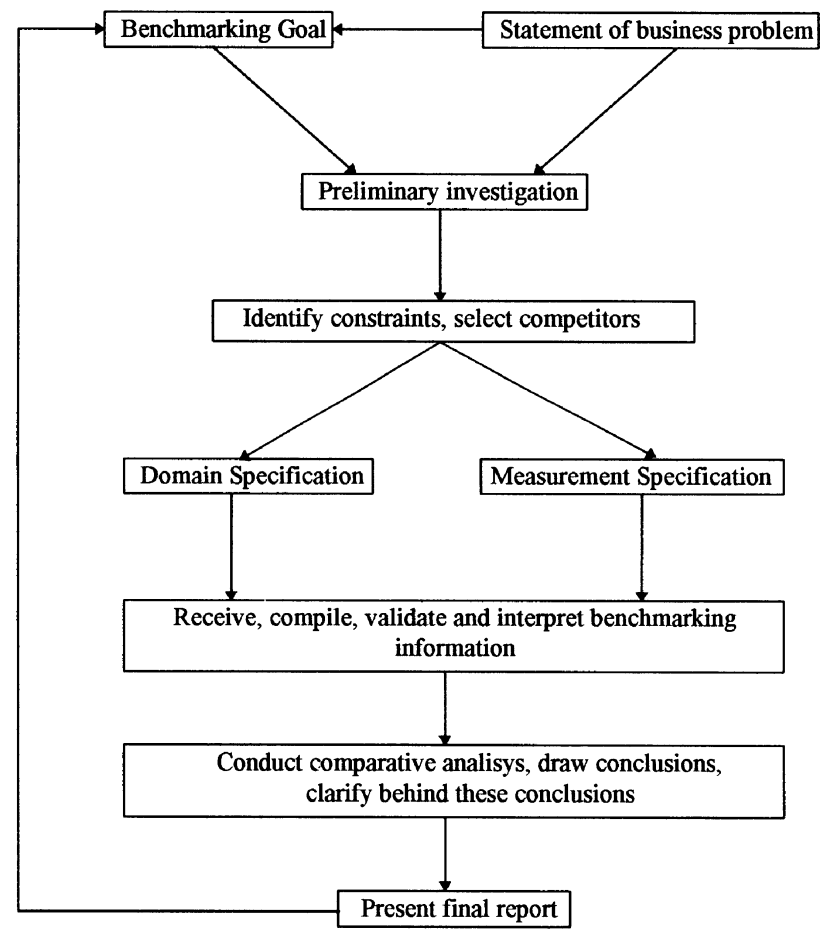

Figure 1 Designing and using software benchmarks. 
The procedure involves eight steps:

1. State the goal. Formulate the business problem and identify decisions which would be made on the base of software benchmarking results

2.Identify constraints. Establish the software-hardware environment where the benchmark should be run. Determine deadlines and allocate resources. Identify benchmarking information sources, types of reportable information, report forms.

3. Select competitors. Choose products of a certain software class according to the goal and the constraints identified.

4.Specify the software benchmark. Define two major benchmark components - the domain specification and the measurement specification. The first one describes completely the software product aspects (granularity) the benchmark should deal with and the second specification establishes the relevant software product characteristics to be measured. It also describes all expected value ranges of all characteristic metrics.

5.Develop a measurement plan. Implement the measurement specification by constructing a set of measured functions. Carry out the measurement, e.g., assign actual values to characteristics involved in benchmarking.

5.Interpret results. Establish rules for using the benchmarking results to solve the business problem formulated.

6.Validate the benchmark. Check the benchmark's pertinence, functional coverage, understandability, ease of use, interpretability and reproducibility.

7.Compare the software products. Conduct analysis and assessment on the base of the benchmarking information. The analysis implies the comparison of target products against a preliminary established model, which serves as benchmark, and the assessment means a study of a set of products so as to rank them with respect to their capability of supporting a predefined objective (Maneva et al., 1994).

8.Develop documentation. Report the results of each stage of the procedure.

\section{COMPUTER-AIDED SOFTWARE BENCHMARKING}

The productivity of the software benchmark investigator can be enhanced by means of a software tools carrying out much of the drudge work associated with benchmark design and use. Thus, computer-aided software benchmarking is the term we introduce to refer to the support of the benchmark investigator using software tools. These tools should involve the following three capabilities: benchmarking situation modelling, integrated information handling, and activity support (reflecting the active aspects in the procedure for benchmark design and use). The aim of situation modelling facilities is to represent the benchmarking universe of discourse in the terms of a certain information model. Integrated information handling is needed to provide the data and knowledge flow throughout the procedure. Finally, the activity support refers to the analysis and the assessment in software benchmarking. It also provides the benchmark investigator with methods for software product comparing and ranking.

\section{SOFTWARE BENCHMARK DESIGN AND USE THROUGH MIC}

To put the notion of computer-aided software benchmarking into the practice we have developed the prototype system MIC (Daneva, 1994). It has been designed to compare software objects (products, processes, resources, software engineering and marketing items) by means of multicriterial methods. The system involves a combination of logical reasoning 
procedures and analytic/algorithmic procedures together with a modularized rule base and a data base. The rule base, the data base and the reasoning procedures were implemented by means of the software package GURU, a tool suitable for intelligent systems development, and the algorithmic procedures were implemented in $\mathrm{C}++$.

Logically, the system comprises two main modules, that provide the construction of benchmarking models and activity support, respectively. Next we discuss the methodology used in each of these components.

\subsection{Methodology}

\section{Applying Modelling Technique to Software Benchmarking}

For conceptualizing the universe of discourse an object-oriented information model has been proposed (Daneva, 1994). We have extended the concept of semantic data modelling (Farmer et al., 1985) by introducing the notion of treating both data and knowledge items in a uniform way. The suggested model is anchored on six key concepts: class, role, simple and composite attribute, function and heuristic. To define them, a set of primitive terms (Table 1) is used.

Table 1. Primitive terms used for concept definitions

\begin{tabular}{ll}
\hline Notation & Meaning \\
\hline\{\} & Set \\
Name & String \\
Domain, Range & Type or Role \\
Restriction & Element of the set \{Single, Multi, Var, Const $\}$ \\
Object & Abstraction unite \\
Body & Algebraic expression \\
Implication & Rule in the form: Algebraic expression $\rightarrow$ Algebraic expression \\
Constraint & Function or Heuristic \\
\hline
\end{tabular}

Next, the modelling concepts are specified:

- A Class is an ordered 5-tuple: (Name, $\{$ Object\}, \{Role\}, \{Simple Attribute\}, \{Function\}).

- A Role is an ordered 6-tuple:

(Name, Function, \{Object\}, \{Simple Attribute\}, \{Role\}, \{Function\}).

- A Simple Attribute is an ordered 5-tuple:

(Name, Domain, Range, \{Restriction\}, \{Constraint\}).

The simple attributes can be multi-valued (Multi) and single-valued (Single), also their values can be changeable (Var) or constant (Const).

- A Composite Attribute is an ordered pair: (Name, \{SAttribute\}).

- A Function is an ordered 4-tuple: (Name, Domain, Range, Body).

Functions are used for constructing roles (IS-A and PART-OF hierarchies), defining nested functions and computing values. The Body of a function is an algebraic expression built by using operations $\left(+,-,{ }^{*}, l\right.$, etc.) and the predefined functions CARDI (returning the cardinal number of a set), SUMA (computing the sum of the element of a set), EXE (executing a function), APPLY (returning values of a selected attribute), MAKEVAL (assigning value to an attribute), X (denoting an object-argument for EXE operator), and INFER (inferring a heuristic).

- A Heuristic is an ordered 4-tuple: (Name, \{Domain\}, \{Range\}, \{Implication\}). The Heuristics are aimed at defining nested heuristics and inferring attribute values.

A simple example of how to specify a benchmarking situation in the terms of the model is given in Figure 2. Let us benchmark Documentation Retrieval Service Software by means of Full Text Document Benchmark defined by DEC experts as follows (Gray, 1993): 
Price/Performance $=$ Five Year Cost of Ownership / Throughput Throughput $=$ Search Transactions Completed per Minute* Database Size in Partition

We consider four software products attributes: Brand, Overall Quality, Workload Search Expression Size and Transaction Mix. Next, the class Benchmark is characterized by the attributes: Name, Value, Min, Max and Subordinate.

CLASS: Doc_Retrieval_Service_Software

INSTANCES: [list of references to software products of the studied class]

S_ATTRIBUTE: (Brand, string, \{Single, Const $\},\{\}$ )

(Quality, string, \{Single, Var $\},\{\mathrm{H}\}$ )

(Workload_Search_Expression_Size, integer, \{Single, Const $\},\{\}$ )

ROLE: ().

(Transaction_Mix, integer, \{Single, Const\}, \{\} ).

FUNKTION: 0 .

HEURISTIC: ().

END.

CLASS: Benchmark

INSTANCES: [list of references to benchmarks]

S_ATTRIBUTE: (Name, string, \{Single, Const\}, \{\})

(Value, real, \{Single, Const $\},\{\mathrm{Hl}\}$ )

(Min, real, \{Single, Const\}, \{\})

(Max, real, \{Single, Const\}, \{\})

(Subordinate, Benchmark, \{Multi, Const\}, \{\}).

ROLE: (Elementary, F1, \{\}$,\{\},\{\}$ ).

FUNKTION: (F1, bool, CARDI(APPLY(Subordinate, $X$ )) $=0$ ).

HEURISTIC: (H, Doc_Retrieval_Service_Software,

APPLY $($ Name,$X)=$ "Performance" and APPLY(Value, $X)>0.9 \rightarrow \operatorname{MAKEVAL(Quality,~} X$,"high"),

APPLY $($ Name,$X)=$ "Performance" and APPLY(Value, $X)<0.2 \rightarrow$ MAKEVAL(Quality, $X$, "low")\})

$(\mathrm{Hl}$, Benchmark, $\{$ APPLY (Name, $\mathrm{X})=$ "Performance" $\rightarrow$

MAKEVAL(Value,X,EXE (*, APPLY (Value, APPLY (Subordinate, X)))),

APPLY (Name, X)! ="Performance" $\rightarrow$

END.

MAKEVAL(Value,X,EXE (/, APPLY (Value, APPLY (Subordinate, X))))\}).

Figure 2 A specification of benchmarking situation for Documentation Retrieval Software.

The function F1 defines which benchmark can be elementary. The heuristic $\mathrm{H}$ captures rules for interpreting the performance benchmark. It specifies the proportionality between the performance measure and the overall software quality. Finally, the heuristic $\mathrm{H} 1$ checks the benchmark's name and prescribes the way of computing the benchmark value. The last can be calculated either by multiplying the subordinate benchmarks, or by dividing them.

\section{Software Product Comparing}

We suggested to use two multicriterial approaches for software product comparing. Anderson's algorithm (Anderson, 1989) was selected for software product assessment. It fits excellently with this problem, but is possesses some peculiarities which impede the support of benchmarking analysis (Daneva, 1994). Thus, to resolve the situation we proposed the $Q R$ approach. To present it we introduce the following definitions: let $n$ be the number of the 
examined products and $\boldsymbol{m}$ - the number of the characteristics considered. Let $\boldsymbol{E}_{(n X m)}$ represent a product-characteristics matrix, each element $e_{i j}$ of which is the evaluation of the $i$ th product in regard to the $j$ th characteristic. Let $\boldsymbol{w}_{(m)}$ be a vector corresponding to the importance weights assigned to the characteristics.

The algorithm $\mathrm{QR}$ involves the following steps:

Step.1.Define an anchored model (standard, benchmark). This is an abstract representation of a software product with artificially constructed attribute values which must be achieved or avoided (Maneva et al., 1994). Let the elements of the vector $t_{(m)}$ describe the anchored model on the characteristics considered.

Step.2.Determine the numbers tplus and tminus given as follows:

tplus $=\max \left(e_{i k}-t_{k}\right)$, where $e_{i k}>t_{k}$

tminus $=\max \left(t_{k}-e_{i k}\right)$, where $e_{i k}<t_{k} \quad i=1, \ldots, n, \quad k=1, \ldots, m$.

There are four cases to be considered:

- values are assigned to both the numbers tminus and tplus;

- a value is assigned to the number tminus, but not to the number tplus;

- a value is assigned to the number tplus, but not to the number tminus;

- values can not be assigned to both the numbers tminus and tplus.

In the last case the algorithm stops. In the other cases it continues.

Step3. For each row $i$ of the matrix $\boldsymbol{E}_{(n X m)}$ we build the set $S$ and calculate the elements $\sup _{i}$ of the vector $\sup _{(n)}$ and $\inf _{i}$ of the vector $\inf _{(n)}$, as follows:

$$
\begin{aligned}
& S=\left\{k \mid e_{i k}>=t_{b} k=1, \ldots, m, i=1, \ldots n\right\} \\
& \sup _{i}=\sum_{k \in S} w_{k}^{*}\left(e_{i k}-t_{k}\right) / \sum_{i=1} w_{k}^{*} t p l u s \\
& \text { inf }_{i}=1-\sum_{k \notin S} w_{k}^{*}\left(t_{k}-e_{i k}\right) / \sum_{i=1} w_{k}^{*} \text { tminus, where } i=1, \ldots, n \text {. }
\end{aligned}
$$

The elements of the set $S$ are the characteristics for which the evaluation received by the $i$ th product is equal to or greater than the evaluation of the anchored model. Next, the element $\sup _{i}$ shows the extent to which the $i$ th product exceeds the anchored model. In contrast, the element inf $f_{i}$ is a measure which indicates the extent to which the $i$ th product is inferior to the model. The greater the elements $\sup _{i}$ and $\inf _{i}$, the more likely that the $i$ th product exceeds the anchored model.

Step4. Define the sets $R_{l i}$ and $R_{2 i}$ for each software product:

$R_{1 i}=\left\{j \mid \sup _{i}>=\sup _{j}, j=1, \ldots, n\right\}$

$R_{2 \bar{\Gamma}}=\left\{j \mid\right.$ inf $\left.\left._{i}\right\rangle=\inf _{j}, j=1, \ldots, n\right\}$, where $i=1, \ldots, n$.

Determine the cardinal numbers $r_{l i}$ and $r_{2 i}$ of the sets $R_{l i}$ and $R_{2 i}$, respectively. The numbers show how many products exceed the $i$ th software package. The comparison is with respect to the elements of the vectors $\sup _{(n)}$ and $i n f_{(n)}$.

Step.5.Calculate the bellow defined cumulative measure com for each product in order to capture the differences across the products - on the one side, and those between the products and the anchored model, on the other side: 
com $_{i}=a l f a{ }^{*} r_{1 i} / n+(1-a l f a){ }^{*} r_{2 i} / n$, where $i=1, \ldots, n, 0<=a l f a<=1$.

The element $\operatorname{com}_{i}$ of the vector $\operatorname{com}_{(n)}$ represents the extent to which the $i$ th product exceeds both the anchored model and the other products examined.

Step.6.Determine the overall quality rating (oqr) for each software product on the base of elements of the vectors $\sup _{(n)}, \inf _{(n)}$, and $\operatorname{com}_{(n)}$. The $o q r$ is considered in dependence on predefined weights corresponding to the contribution of each measure to the overall rating. Let $a w_{1}, a w_{2}$ and $a w_{3}$ represent the weights chosen for the vectors sup, inf and com, respectively. The numbers $a w_{1}, a w_{2}$ and $a w_{3}$ should be in the interval $[0,1]$, and its sum has to equal to 1 . The oqr for the $i$ th product is given by:

$o q r_{i}=a w_{1}{ }^{*} \sup _{i}+a w_{2}{ }^{*} \inf _{i}+a w_{3}{ }^{*} \operatorname{com}_{\dot{p}}$, where $i=1, \ldots, n$.

Step.7.Rank the products according to their oqr. The rank is a number between 1 and $n$, where 1 means excellent and $n$ means poor. The product with the greatest oqr is the best.

\subsection{Application}

Next, we give examples of how the system MIC could be used. We consider two software benchmarking problems described in the terms of objectives, competitive software products, comparative characteristics and importance weights.

\section{CASE STUDY I:}

Objective: To determine the appropriateness of CASE tools to build a Quality Management System according to the requirements of the standard ISO 9000.

Software Class: CASE tools.

Software Characteristics: The analyzed features are given in Table 1(Herzwurm, 1994).

Software Products: CASE tools available on German market (Herzwurm, 1994).

Table 1 Characteristics and importance weights for CASE tools

\begin{tabular}{llll}
\hline Characteristics: & Weights: & Characteristics: & Weights: \\
\hline Team work & 1 & Documentation Development & 9 \\
Openness & 4 & Quality Assurance & 9 \\
Analysis Support & 3 & Project Management & 5 \\
Design Support & 3 & Configuration Management & 7 \\
\hline
\end{tabular}

Two cases have been studied - with equal characteristic weights and with weights, defined by researchers from the University of Köln (Herzwurm, 1994). QR approach was used. The anchored model was constructed by using the maximal software evaluations on the characteristics considered. The results are presented in Table 2 . 
Table 2 Ranking of CASE tools

\begin{tabular}{lll}
\hline CASE tools: & Ranking I: & Ranking II: \\
\hline Maestro II & 1 & 1 \\
Teamwork & 3 & 2 \\
I-Case Yordon & 2 & 3 \\
Innovator & 4 & 4 \\
SDW & 6 & 5 \\
I-Case OMT & 7 & 6 \\
ADW & 5 & 7 \\
Systems Engineering & 8 & 8 \\
Excelerator II & 10 & 9 \\
DDB CASE & 11 & 10 \\
IEF & 9 & 11 \\
case/4/0 & 12 & 12 \\
\hline
\end{tabular}

\section{CASE STUDY II}

Objective: To rank software vendors.

Software Class: Data Base Management Systems (DBMS).

Software Characteristics: The analyzed features are given in Table 3 (Datapro, 1993).

Software Products: Ingres, Oracle, Sybase, Focus, Informix (Datapro, 1993).

Table 3 Characteristics and importance weights for data base software

\begin{tabular}{llll}
\hline Characteristics: & Weights: & Characteristics: & Weights: \\
\hline Price/Performance & 8 & Quality of Output & 6 \\
Reliability & 7 & Understandability & 8 \\
Ease of Learn & 10 & Userfriendness & 9 \\
Flexibility & 4 & OS Compatibility & 3 \\
\hline
\end{tabular}

Two cases have been studied - with equal characteristic weights and with weights defined by means of the system MIC. The software packages have been ranked by using $Q R$ approach. The anchored model was constructed by using the average values on the characteristics analyzed. The results are presented in Table 4.

Table 4 Ranking of data base software

\begin{tabular}{lll}
\hline$D B M S:$ & RankI: & RankII: \\
\hline Informix & 1 & 1 \\
Sybase & 2 & 3 \\
Ingres & 3 & 2 \\
Focus & 4 & 5 \\
Oracle & 5 & 4 \\
\hline
\end{tabular}

\section{CONCLUSIONS AND FUTURE DIRECTIONS}

Software practitioners, marketers and academicians have all emphasized different software performance issues and have used different approaches to solve particular software benchmarking problems.

In this paper, we have developed a holistic view of software benchmarking which has not been previously reported. The key research contribution of our work is the presentation and 
the implementation of an unifying approach to software benchmark design and use. The focus of the approach is on what steps a software benchmarking process consists of and how they are to be performed. We have proposed a model for representing benchmarking situations, an algorithm for software product ranking and a prototype intelligent software system MIC. Moreover, by applying the approach to CASE-tools and data base assessment, the power of its features to capture subtle benchmarking issues are fully illustrated.

Our investigation suggests a variety of topics for further research referring to the gap between current product-centered software benchmarking and the process dimension of software engineering. The future work will be concentrated on:

- testing the adequacy of the approach to benchmark software processes;

- relating software benchmarking and software process re-engineering;

- developing practical algorithms so as to contribute towards a set of tools to aid in the systematic software process benchmarking.

\section{REFERENCES}

Anderson, E. (1989) Heuristics for software evaluation and selection. Software Practice and Experience, 8, 707-17.

Ghezzi, C., Yazayeri, M., Mandriorli, D. (1991) Fundamentals of software engineering. Prentice-Hall, New Jersey.

Gray, J. (1993) Benchmarking handbook for database and transaction processing systems. Morgan Kaufman Publ., California.

Daneva, M. (1994) Knowledge-based approach to software marketing modelling and support. PhD Dissertation, Institute of Mathematics with Computing Center, Sofia.

Datapro Research Corporation, Report on Software/Computer System, Series Software.

Farmer, D., King, R., Mayers, D. (1985) The semantic database constructor. IEEE Transactions on Software Engineering, 11, 1985, 583-90.

Herzwurm, G., Hierholzer, A., Kung, M. (1994) The appropriateness of the conventional and object-oriented CASE-tools to construct a Quality Management Systems according ISO 9000. Information Management, 3, 72-6.

Maneva, N., Daneva, M., Petrova, V. (1994) Benchmarking in Software Development, in Benchmarking - Theory and Practice, IFIP Working Group 5.7, Trondheim.

McDonell, K. (1995) Benchmarking frameworks and tools for modelling the workload profile. Performance Evaluation, 1, 23-42.

Porscher, T. (1995) Benchmarking the POM PC compiler on the Connection Machine CM26. Future Generation Computer Systems, 11, 19-26.

I thank Ralf Heib for comments and suggestions that led to the improved presentation of this paper. This research is funded by German Academic Exchange Service under grant A/94/10247 and Ministry of Science and Education under the contract I-406.

Maya Daneva received her M.Sc. degree in Computer Science from the University of Sofia, 1991. PhD Dissertation, Institute of Mathematics, BAS, Sofia, 1994. Currently with the Institute of Business Information Systems, University of Saarland.

Her scientific interests include: Software Engineering, Software Benchmarking, Software Marketing Modelling, Knowledge-Based Marketing Systems. 